\title{
"Estudio exploratorio descriptivo de la práctica profesional de los terapeutas ocupacionales en el sistema educativo regular que apoyan los procesos de integración educativa en las Regiones Quinta y Metropolitana durante el año 2005."
}

Araya Orellana, Esperanza ${ }^{[1]}$; Araya Saavedra, Catalina ${ }^{[1]}$; Caro Vines, Pamela ${ }^{[1]}$; Espinosa Valderrama, Alejandra ${ }^{[1]}$ y González Godoy, Claudia ${ }^{[1]}$; TO guía: Goudeau Radical, Dalila ${ }^{[2]}$

\section{- Resumen}

Objetivos: Conocer características demográficas, de formación profesional, y características de la práctica profesional de Terapeutas Ocupacionales que apoyan los procesos de integración educativa al Sistema Educativo Regular, en las regiones Quinta y Metropolitana, durante el año 2005.

Metodología: Estudio no experimental, transversal y descriptivo, muestra no probabilística, constituida por 41 Terapeutas Ocupacionales. Se construye el instrumento "Cuestionario para Terapeutas Ocupacionales en Educación Regular", aplicado durante el 12 de diciembre de 2005 y 06 de enero de 2006. Análisis y tratamiento de datos según estadística descriptiva; muestra se subdivide en Terapeutas Ocupacionales que trabajan en Proyectos de Integración Escolar (PIE) y estrategias complementarias (SIN PIE).

Resultados y conclusiones: Existen Terapeutas Ocupacionales apoyando procesos de integración educativa, con diferencias en la práctica profesional entre ambas estrategias. Se desempeñan en Quinta Región y Metropolitana, egresan de Universidad de Chile y Universidad de Playa Ancha de Ciencias de la Educación, se perfeccionan en distintas áreas, la mayoría no realiza publicaciones. Diferencias en condiciones laborales y población intervenida; similitudes en nivel educativo de los estudiantes que reciben el apoyo y marcos teóricos utilizados. Ambos grupos evalúan distintos ámbitos del desempeño ocupacional, preferentemente con instrumentos no estandarizados; incluyen actividades en el contexto de desempeño escolar físico y social, modalidad de trabajo individual y grupal, y otras funciones; trabajan mayormente en equipo multiprofesional. Terapeutas Ocupacionales de ambas estrategias apoyan integración y realizan acciones inclusivas.

Palabras claves: Práctica profesional, proceso de integración educativa, desempeño ocupacional, contexto de desempeño ocupacional. 


\begin{abstract}
- Abstract
Goals: To determjne demographic characteristics, professional training and professional practice characteristics of Occupational Therapists that support scholar integration processes to the Regular Education System in the Fifth and Metropolitan Regions, throughout 2005.

Methodology: Non-experimental, cross sectional and descriptive study, nonprobabilistic sample, composed by 41 Occupational Therapists. The "Questionaire for Occupational Therapists in Regular Education" was developed, which was applied during December 12, 2005, and January 6, 2006. Analysis and data processing were carried out according to descriptive statistics; sample is subdivided in Occupational Therapists who work in Scholar Integration Projects (SIP) and complementary strategies (without SIP).

Results and conclusions: There are Occupational Therapists supporting educational integration processes, with differences in the professional practice between both strategies. They work in the Fifth and Metropolitan regions, graduated from Universidad de Chile and Universidad de Playa Ancha de Ciencias de la Educación, they perform further training in different areas, most of them do not make publications. Differences in laboral condition and in the intervening population are present; similarities in the educational level of the students and in the theorical frameworks used. Both groups evaluate different field of the occupational performance, especially non-standardized instruments; they include activities in the physical and social context of scholar performance, individual and group work modality, and other functions; they work mainly in multiprofessional teams. Occupational Therapists of both strategies support the integration and make inclusive actions.
\end{abstract}

Key words: Professional practice, scholar integration process, occupational performance, occupational performance context.

\footnotetext{
${ }^{[1]}$ Terapeuta Ocupacional, Licenciada en Ciencias de la Ocupación, Universidad de Playa Ancha de Ciencias de la Educación

${ }^{[2]}$ Magíster en Pedagogía Universitaria (C) Universidad de Playa Ancha de Ciencias de la Educación; Terapeuta Ocupacional, Universidad de Chile (1971)
} 


\section{Introducción}

A fines de la década de los ' 50 surge el principio de normalización, que provoca un cambio en la valorización de la persona con discapacidad, poniendo el énfasis en los procesos de integración y participación social. En el ámbito educativo, esto significó un cambio en la forma de concebir la enseñanza, caracterizado por el abandono paulatino del enfoque rehabilitador para centrarse en un marco principalmente educativo, el que los estudiantes con y sin discapacidad participan juntos en procesos de aprendizaje.

Bajo este principio, en el año 1978 en el Reino Unido, se publica el Informe Warnock ${ }^{(\underline{1})}$, en el que se plantea por primera vez el principio de integración escolar, señalando que los fines de la educación son los mismos para todas las personas; además, se propone el concepto necesidades educativas especiales (NEE) que reemplaza al de discapacidad.

En el año 1989 la Organización de las Naciones Unidas, publica la Convención de los Derechos del $\mathrm{Niño}^{(\underline{2})}$. En ella se reconoce a los niños como sujetos de derecho, siendo uno de estos la educación, que debe ser eximida de discriminación.

En el año 1994 se publica la Declaración de Salamanca( ${ }^{(\underline{3})}$ cuya finalidad es promover la educación para todos. Plantea que se debe fomentar y facilitar la participación de los padres en este proceso, potenciar la participación de la comunidad internacional en el intercambio de experiencias y en la defensa del enfoque de escolarización integradora.

En Chile, el primer paso hacia la integración escolar es el Decreto Supremo de Educación No $490^{(4)}$, el cual establece normas técnicas y administrativas para integrar a estudiantes con discapacidad en establecimientos educacionales comunes.

En el año 1994 se promulgada la Ley No 19.284/94, Sobre La Plena Integración Social de las Personas con Discapacidad ${ }^{(5)}$, la que en el título IV, capítulo II considera el acceso a la educación. Plantea que los establecimientos públicos y privados del sistema educativo regular deberán permitir y facilitar la permanencia y progreso de los alumnos con NEE en todos los niveles del sistema; los equipos multiprofesionales del Ministerio de Educación entregarán informes que definan la necesidad de la persona con discapacidad y por lo tanto la modalidad, el tiempo y el establecimiento educacional adecuado; además, establece que las escuelas especiales deberán proveer recursos especializados y asesorías a todos los niveles educativos.

En el año 1998 se publica el Decreto Supremo de Educación No 01/98(ㅁ) que norma lo establecido en la Ley 19.284, deroga el Decreto 490, siendo el vigente en la actualidad. Este documento establece que "el proceso de integración educativa consiste en educar niños y niñas, jóvenes y adultos con y sin discapacidad durante una parte o la totalidad del tiempo en establecimientos de educación común, el que comenzará preferentemente en el periodo preescolar pudiendo continuar hasta la educación superior"(ㅁ); también define el aula de recursos como el espacio funcional para satisfacer las necesidades de los alumnos integrados. Además establece el beneficio de subvención y señala cuatro opciones de integración educativa, que van desde la plena integración a otras opciones más restrictivas.

En el año 1996, el Sistema Educativo Nacional pone en marcha la Reforma Educacional que impulsa cambios hacia un sistema más amplio y modernizado, en el que las prácticas pedagógicas se fundamentan en principios del Humanismo y 
Constructivismo; en este proceso se incluye la Educación Especial, es así como en el año 2003 se crea la Comisión de Expertos de Educación Especial. Un año más tarde se presentan los resultados de dos estudios que permiten fundamentar la implementación de una nueva política de educación especial.

El Estudio a Nivel Muestral de la Calidad del Proceso de Integración Educativa(ㄱ) , en relación con los equipos profesionales, determina que el trabajo en equipo es minoritario y de modalidad preferentemente interdisciplinaria, la forma de organización es principalmente con equipos multiprofesionales comunales que itineran de un establecimiento a otro, EL rango de horas trabajadas varía entre 2 dos a 15 horas por escuela, con tendencia a que el apoyo ocurra en el aula de recursos más que en el aula regular.

El año 2004 se realiza en Chile el Primer Estudio de la Discapacidad(홍, el que concluye que, a nivel nacional: una de cada dos personas con discapacidad no ha completado su educación básica, una de cada 10 ha cursado la enseñanza media completa y solo una de cada veinte ha logrado acceder a la educación superior

En el año 2005 se presenta la nueva Política Nacional de Educación Especial ${ }^{(\underline{9})}$ en la que se destaca: aumento de la cobertura; señala que los factores de contexto pocas veces son considerados en la respuesta educativa, establece que se debe garantizar la máxima participación en el currículo común y en las actividades escolares; reconoce la inexistencia de unanimidad en las condiciones de evaluación de necesidades educativas y que el apoyo debe estar orientado mayormente al profesor más que a los alumnos.

En cuanto a Terapia Ocupacional, existen antecedentes internacionales de la participación de este profesional en la educación común (EE.UU, Colombia, Reino Unido), reconocida en la normativa vigente, la población beneficiada incluye a personas con y sin discapacidad, profesores y familia, la práctica profesional se lleva a cabo en equipo de profesionales, se sistematiza la información y se realizan publicaciones. Al menos en EE.UU., esto ha permitido una formación mas especifica en esta área.

El Colegio de Terapeutas Ocupacionales de Chile, AG. ha elaborado dos documentos en respuesta a las nuevas propuestas en educación especial. En ellos comparte con el Ministerio de Educación la visión integral de este profesional, la necesidad aumentar la participación de la comunidad y contextualizar las intervenciones, considerando tanto el ambiente físico como el social. Además, en el Perfil por Competencias de Terapia Ocupacional ${ }^{(10)}$, elaborado para el Ministerio de Salud, se reconoce la educación como una de sus áreas de práctica profesional.

Para explicar el Sistema Educativo Regular como área de práctica de Terapia Ocupacional, el marco teórico considera los fundamentos humanistas y constructivistas de la Reforma Educacional y los conceptos básicos de Terapia Ocupacional, según las definiciones del documento "Marco de Trabajo para la Práctica de Terapia Ocupacional: Dominios y Práctica"(11). Además, se utiliza el Modelo de Ocupación Humana, que por sus características sistémicas, permite comprender cómo el ambiente demanda o facilita comportamientos ocupacionales.

Estos ámbitos se relacionan estableciendo como parámetro la interacción entre persona, ambiente y actividad. En el ámbito educativo, la interacción entre estos factores, permite el aprendizaje de contenidos y habilidades funcionales, preparando al 
estudiante para la participación en nuestra sociedad; esto se relaciona con el principal objetivo de Terapia Ocupacional, que pretende lograr el desempeño funcional para favorecer la participación en ocupaciones en distintos contextos

En base a lo antes señalado, la práctica de Terapia Ocupacional en el Sistema Educativo Regular se centra en el desempeño ocupacional del rol de estudiante, cuya principal ocupación es la participación en procesos de aprendizaje significativos en el contexto escolar. De esta manera el Terapeuta Ocupacional contribuye al desempeño de este rol, colabora en el mejoramiento de procesos de aprendizajes y favorece la adaptación al contexto escolar, según ámbitos y procesos profesionales.

\section{- Planteamiento del problema}

Existen antecedentes del escaso acceso, mantención y egreso de personas con discapacidad en el sistema educacional lo que plantea un nuevo desafío a las políticas nacionales: respetar del derecho de todas las personas a la educación. Como una manera de dar solución a este problema, se propone la implementación de los PIE en establecimientos de educación regular; junto con ellos, nacen estrategias complementarias.

Para la puesta en marcha de los PIE se establece la necesidad de trabajo en conjunto con profesionales de otras áreas de intervención, sin embargo, no establece quienes son dichos profesionales. En la literatura nacional existen dos documentos que señalan los aportes del T.O en el área de educación, ambos provienen del Colegio de Terapeutas de Chile, estos fueron elaborados por un grupo reducido de profesionales, lo que no permite generalizar los resultados.

Entonces, nos encontramos con el problema del escaso conocimiento de la Práctica Profesional del Terapeuta Ocupacional en el Sistema Educativo Regular. Esto afecta la conformación del equipo de profesionales, pues no se cuenta con un profesional que analice la forma en que el ambiente facilita o dificulta el desempeño y tampoco con quien proponga los ajustes necesarios en el ambiente físico y/o social; lo que repercute en sobrecarga laboral del docente y reducción de oportunidades de mejorar el desempeño ocupacional del rol de estudiante. Esta situación incrementa las dificultades para el logro de la integración y la adquisición del aprendizaje.

En base a lo antes señalado, surgen dos preguntas de investigación: ¿Hay Terapeutas Ocupacionales (TTOO) apoyando el proceso de integración educativa?, si es así, ¿Cuáles son las características de su práctica profesional? 
Objetivos del estudio

1. Conocer características demográficas y de formación profesional de los TTOO que apoyan los procesos de integración educativa en las regiones Quinta y Metropolitana.

a. Determinar número de TTOO que apoyan estos procesos

b. Determinar distribución geográfica

c. Conocer procedencia universitaria

d. Caracterizar experiencias, perfeccionamiento a los que han accedido y/o publicaciones realizadas

2. Establecer características de práctica profesional de los TTOO que apoyan los procesos de integración educativa en las regiones Quinta y Metropolitana.

a. Describir las condiciones de trabajo en las que se desempeñan los TTOO

b. Caracterizar la población en la que intervienen

c. Determinar los fundamentos teóricos que sustentan la intervención

d. Conocer las estrategias de intervención

e. Caracterizar el equipo profesional

\section{- Justificación del estudio}

Los resultados de estudios actuales hacen evidente la necesidad de trabajar por la equidad y calidad de la educación para todas las personas, señalando como fundamental contar con recursos humanos calificados que realicen un trabajo colaborativo con los profesionales docentes.

El conocimiento de la práctica profesional de los TTOO apoyando los procesos de integración educativa, es un aporte significativo en: La búsqueda de estrategias adecuadas para solucionar los problemas que actualmente presentan los PIE, contribuyendo al fortalecimiento de los equipos de apoyo, aportando al trabajo transdisciplinario; el estudio posee un valor social que se relaciona directamente con la necesidad de un cambio cultural hacia la aceptación de la diversidad y un valor teórico que radica principalmente en la necesidad de sistematizar información que permita establecer un fundamento teórico sobre la práctica profesional actual. Finalmente, se espera aportar desde la profesión al logro de la equidad y calidad de la educación.

\section{- Metodología}

Diseño de investigación es no experimental transversal descriptivo, en el marco de un estudio de tipo exploratorio descriptivo. La muestra utilizada es no probabilística, delimitada el siguiente criterio de selección: TTOO que apoyan procesos de integración educativa al sistema educativo regular desde PIE y/o experiencias complementarias, en las regiones Quinta y Metropolitana, durante al menos un mes del año 2005.

La selección de la muestra se realizó según estrategias de convocatoria de sujetos tipos y sujetos voluntarios a partir de gestión de las propias investigadoras, publicación 
en página Web del Colegio de Terapeutas Ocupacionales de Chile, convocatoria en Congreso Latinoamericano de Integración Sensorial y Congreso de Profesionales no Docentes de Educación, además de las referencias de TTOO que participan en el estudio. Se contacta a 63 TTOO, de estos, 41 contestan el instrumento, constituyendo la muestra final, lo que corresponde a un $5,18 \%$ del total nacional de TTOO titulados (12).

Para recolectar los datos se construye el instrumento "Cuestionario para Terapeutas Ocupacionales en Educación Regular", en base a cinco variables del estudio: identificación personal, formación profesional, condiciones laborales, características de la población e intervención. El instrumento es sometido a pruebas de validación y confiabilidad, lo que indica consistencia interna, es confiable y es posible su aplicación.

El instrumento está formado por 26 preguntas distribuidas en 5 ítems. Es un cuestionario auto administrado, para contestar en un tiempo de 15 minutos, durante el cual existió la posibilidad de realizar consultas. Se aplica desde el 12 de diciembre del 2005 al 06 de enero 2006.

Se reconocen como principales limitaciones: la escasa literatura e información referente al tema, sobre todo a nivel nacional; dificultad para convocar y contactar a los TTOO, debido a la inexistencia de registros tanto en instancias educativas como de Terapia Ocupacional, disponibilidad de tiempo, sobrecarga laboral y fecha de aplicación.

En el tratamiento de los datos se utilizan medidas de tendencia central y estadísticas descriptivas, los resultados se presentan con muestra final subdivida en TTOO que se desempeñan en PIE y TTOO que trabajan en estrategias complementarias. Cabe señalar que las frecuencias absolutas pueden no corresponder con la cantidad de TTOO que responden el instrumento pues la mayoría de las preguntas permiten marcar más de una opción.

\section{- Resultados}

\section{Objetivo General 1:}

Determinar características demográficas y de formación profesional de los TTOO que apoyan los procesos de integración educativa.

De los 41 TTOO que contestan el instrumento, 15 se desempeñan en PIE y 26 en estrategias complementarias, en ambos grupos la mayoría son de sexo femenino. Con respecto a la formación profesional, la mayoría de los TTOO que se desempeñan en ambas estrategias son titulados de la Universidad de Chile y sólo cinco de la Universidad de Playa Ancha de Ciencias de la Educación.

En cuanto a la región de trabaja, 11 TTOO intervienen en la Quinta región, cuatro de ellos en PIE y siete en estrategias complementarias. Los TTOO que se desempeñan en PIE abarcan cuatro comunas, en tres de ellas: Quillota, Putaendo y Villa Alemana, existe 1 TO por cada colegio; en Viña del Mar existe 1 TO que se desempeña en 14 colegios. 
Los TTOO que trabajan en estrategias complementarias lo hacen en cuatro comunas. En la comuna de Valparaíso existen cuatro TTOO que se desempeñan en institución no ligada a educación y que no especifica cantidad de colegios.

En la Región Metropolitana, intervienen un total de 30 TTOO, 11 de ellos en PIE y 19 en estrategias complementarias. Los que se desempeñan en PIE abarcan 10 comunas, llama la atención que comunas como Puente Alto, Santiago Centro e Independencia cuentan con un TO para 1 colegio y en Pudahuel exista un TO para 11 colegios.

Los TTOO que se desempeñan en estrategias complementarias abarcan una totalidad de 14 comunas. En Las Condes, por ejemplo, intervienen siete TTOO distribuidos en 10 colegios, en Quinta Normal, Pudahuel, Estación Central y San Joaquín los TTOO no especifican cantidad de colegios intervenidos.

Con respecto al tiempo de experiencia laboral, la mayoría de los TTOO que se desempeñan en PIE tienen una experiencia laboral de hasta dos años, lo que concuerda con que la mayoría de los TTOO egresaron entre los años 2001 y 2004 . El mayor porcentaje de los TTOO que se desempeñan en estrategias complementarias indica entre seis y siete años de experiencia laboral en el área.

La mayoría de los TTOO que se desempeñan en ambas estrategias se perfeccionan principalmente en las áreas de educación en Integración Sensorial (ver gráfico 1), y la mayoría de ellos no realiza publicaciones.

\section{Gráfico 1: Distribución porcentual de TTOO según lugar de trabajo y área de perfeccionamiento}

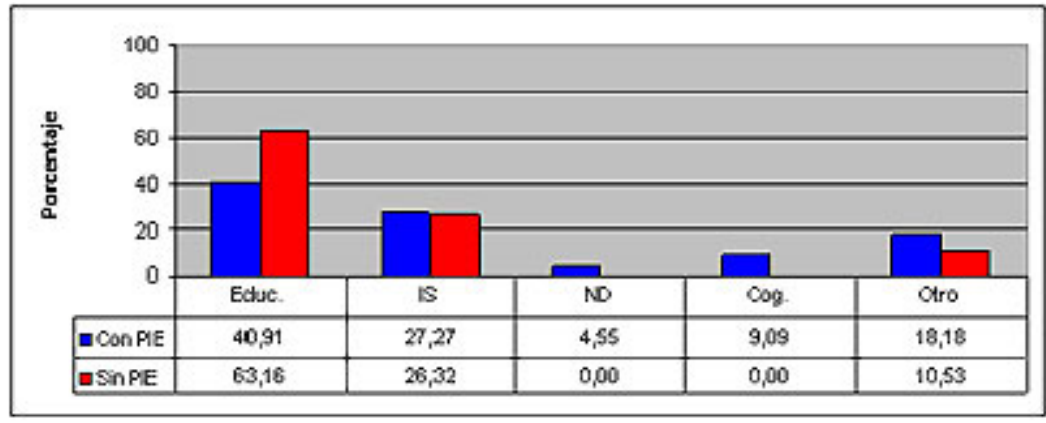

Figura 1: Distribución porcentual de TTOO según lugar de trabajo y área de perfeccionamiento. Educ.: Educación IS: Integración SensorialND: Neurodesarrollo Cog.: Cognitivo.

\section{Objetivo General 2:}

Establecer las características de la práctica profesional de los TTOO que apoyan el proceso de integración educativa.

En cuanto a la institución que contrata, la mayoría de los TTOO que trabajan en PIE son contratados por Corporaciones Municipales, los que se desempeñan en estrategias complementarias son contratados mayoritariamente por otro tipo de institución (ejercicio libre de la profesión, Centros de rehabilitación, Hospitales) 
Respecto a la cantidad de horas semanales y alumnos intervenidos (ver tabla 1), los TTOO que trabajan en PIE entre 13 a más de 42 horas se desempeñan principalmente en establecimientos con financiamiento municipal. Los TTOO que trabajan en ambas estrategias entre 13 y 24 horas semanales, intervienen en establecimientos municipalizados abarcando a una población de más de 50 alumnos realizando principalmente intervenciones psicosociales. Considerando el rango de 31 a 42 horas, los TTOO que trabajan en PIE atienden entre 11 y 40 alumnos en un establecimiento con financiamiento municipal, mientras que los que se desempeñan en estrategias complementarias atienden a una cantidad de alumnos menor en un establecimiento con financiamiento particular.

Tabla 1

\section{Cantidad de alumnos y tipo de financiamiento según cantidad de horas semanales}

\begin{tabular}{|l|l|l|l|l|}
\hline & \multicolumn{2}{|c|}{ PIE } & \multicolumn{2}{c|}{ SIN PIE } \\
\hline Cant. Horas & \multicolumn{1}{|c|}{ No Alumnos } & Tipo financiamiento & No Alumnos & \multicolumn{1}{|c|}{ Tipo Financiamiento } \\
\hline $1-12$ & $1-20$ & Part. subvencionado & $1-40$ & Part/ part. subvencionado \\
\hline $13-24$ & $1-20 /+50$ & Municipal & +50 & Municipal \\
\hline $15-30$ & $11-20$ & Part- subv/Municipal & $41-50$ & Municipal \\
\hline $31-42$ & $11-40$ & Municipal & $1-10$ & Particular \\
\hline
\end{tabular}

Con relación a la población intervenida (ver gráfico 2), los TTOO que trabajan en PIE dirigen su intervención principalmente a profesores, estudiantes con NEE asociadas a discapacidad y sus familias. Los TTOO que se desempeñan en estrategias complementarias atienden principalmente a estudiantes con NEE asociadas o no a discapacidad y en menor porcentaje a familias y profesores. En ambas estrategias el nivel educativo básico es el más intervenido.

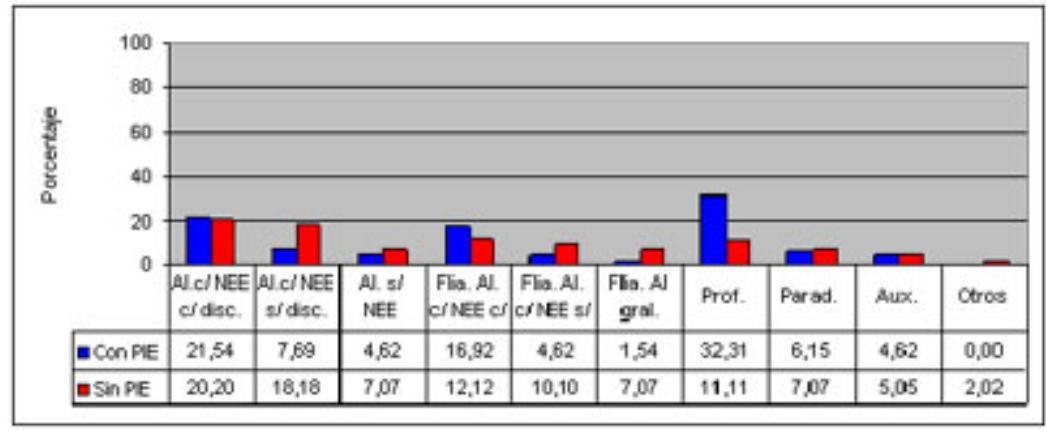

Figura 2: Distribución porcentual de TTOO según lugar de trabajo y población

Al.c/NEE c/disc. : Alumnos con Necesidad Educativa Especial con discapacidad Al.c/NEE s/disc. : Alumnos con Necesidad Educativa Especial sin discapacidad Al.s/NEE : Alumnos sin Necesidad Educativa Especial

Flia. Al.c/NEE C/ : Familias de alumnos con Necesidad Educativa Especial con discapacidad Flia. Al.c/NEE s/ : Familias de alumnos con Necesidad Educativa Especial sin discapacidad Flia.Al Gral : Familias de Alumnos en General

Prof. : Profesores

Parad.: Paradocentes

Aux. : Auxiliares

Otros : Otras personas de la Comunidad Educativa 
En cuanto al tipo de discapacidad de los alumnos (ver gráfico 3), los TTOO que se desempeñan en PIE atienden principalmente a alumnos con trastornos motores y deficiencia mental; por su parte, los que se desempeñan en estrategias complementarias, centran su atención tanto en alumnos con trastornos motores como en aquellos que presentan alteraciones de la relación y la comunicación oral. La información recopilada permite corroborar que los TTOO intervienen a alumnos con discapacidades no consideradas en los PIE, como son las sensorio-integrativa y las psicosociales.

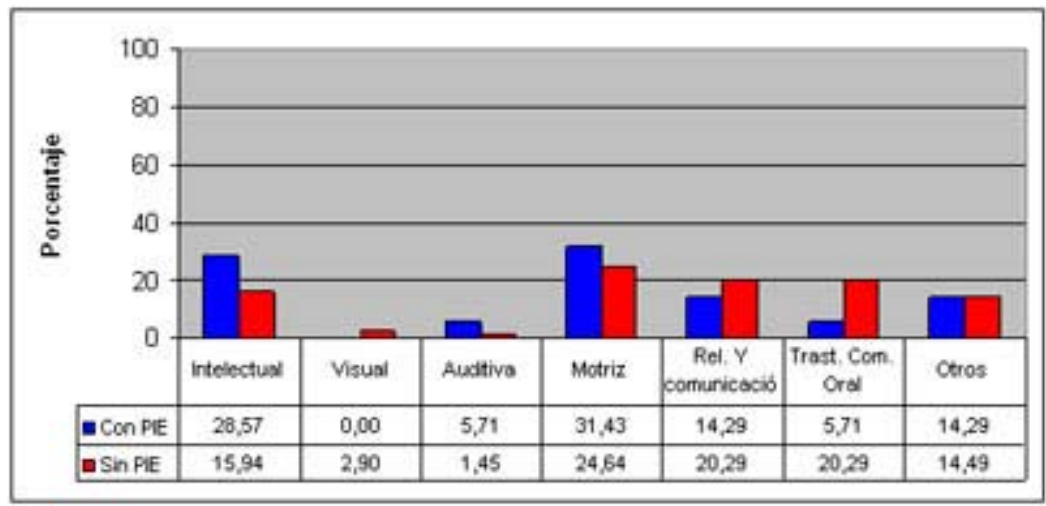

Figura 3: Distribución porcentual de TTOO según lugar de trabajo y tipo de discapacidades de los estudiantes

Rel. Y comunicación: Relación y la Comunicación

Trast.Com.OralTrastorno de la Comunicación Oral.

Otros: Incluye disfunciones psicosociales y trastornos de integración sensorial.

Respecto al marco de referencia utilizado, el más señalado en ambas estrategias es el de Integración Sensorial, esto coincide con el área de perfeccionamiento más frecuentemente mencionado. En cuanto al tipo de evaluación utilizada, los TTOO que trabajan en ambas estrategias usan preferentemente pautas no estandarizadas, siendo la más señalada observación clínica del desempeño ocupacional en el contexto habitual.

Todos los TTOO que participan en el estudio, refieren intervenir en alguno de los componentes del ambiente físico (ver tabla 2) El componente más intervenido en ambas estrategias es el mobiliario, desde el punto de vista de reorganización del espacio, diseño ergonómico y evaluación de necesidad de ayudas técnicas. En cuanto a la infraestructura, los TTOO que trabajan en ambas estrategias centran su intervención en la evaluación del entorno. En cuanto a los objetos los TTOO de ambas estrategias realizan preferentemente diseño y elaboración de adaptaciones. 
Tabla 2

Distribución porcentual de TTOO según tipo de actividad y componentes del ambiente físico

\begin{tabular}{|l|c|c|c||c|r||r|}
\hline \multirow{2}{*}{ ACTIVIDAD } & Infraestructura & \multicolumn{2}{|c|}{ Mobiliario } & \multicolumn{2}{c|}{ Objetos } \\
\cline { 2 - 6 } & PIE & SIN PIE & PIE & SIN PIE & PIE & SIN PIE \\
\hline \hline Evaluación del entorno físico & $37,50 \%$ & $42,80 \%$ & $29,17 \%$ & $32,14 \%$ & $33,33 \%$ & $25,00 \%$ \\
\hline \hline Reorganización del espacio & $23,81 \%$ & $47,62 \%$ & $47,60 \%$ & $38,10 \%$ & $28,57 \%$ & $27,27 \%$ \\
\hline Diseño ergonómico & $23,08 \%$ & $23,81 \%$ & $46,10 \%$ & $38,10 \%$ & $30,77 \%$ & $38,10 \%$ \\
\hline Diseño y elaboración de adaptaciones & $18,18 \%$ & $28,57 \%$ & $40,91 \%$ & $28,57 \%$ & $40,90 \%$ & $42,80 \%$ \\
\hline \hline Eval.de necesidad de ayudas técnicas & $26,67 \%$ & $30,00 \%$ & $46,60 \%$ & $40,00 \%$ & $26,67 \%$ & $30,00 \%$ \\
\hline
\end{tabular}

Con respecto al ambiente social (ver tabla 3), los TTOO de ambas estrategias realizan promoción, principalmente a docentes y técnicos en educación; en cuanto al entrenamiento directo de habilidades funcionales y a la educación, en ambas estrategias la intervención se centra en la familia.

Tabla 3

\section{Distribución porcentual de TTOO según tipo actividad y componente del ambiente social}

\begin{tabular}{|c|c|c|c|c|c|c|}
\hline \multirow[t]{2}{*}{ ACTIVIDAD } & \multicolumn{2}{|c|}{ Promoción } & \multicolumn{2}{|c|}{$\begin{array}{l}\text { Entrenamiento } \\
\text { Habilidades } \\
\text { funcionales }\end{array}$} & \multicolumn{2}{|c|}{ Educación } \\
\hline & PIE & SIN PIE & PIE & SIN PIE & PIE & SIN PIE \\
\hline Docentes y téc. en educación & $37,50 \%$ & $28,57 \%$ & $22,22 \%$ & $30 \%$ & $16,67 \%$ & $28,57 \%$ \\
\hline No docente & $6,25 \%$ & $28,57 \%$ & & $5 \%$ & - & $14,29 \%$ \\
\hline Familia & $25 \%$ & $28,57 \%$ & $55,50 \%$ & $45 \%$ & $58,33 \%$ & $42,86 \%$ \\
\hline Pares & & - & $11,11 \%$ & $10 \%$ & $8,33 \%$ & $7,14 \%$ \\
\hline Grupo Curso & $18,75 \%$ & - & - & $10 \%$ & $8,33 \%$ & 0 \\
\hline Red social & $12,50 \%$ & $14,29 \%$ & $11 \%$ & - & $8,33 \%$ & $7,14 \%$ \\
\hline
\end{tabular}

Respecto al tipo de intervención individual, la más señalada en ambas estrategias se relaciona con la evaluación de ingreso al establecimiento y la detección de NEE, además de tratamiento individual (en menor grado); las actividades se realizan en sala de clases y/o aula de recursos. Los TTOO que trabajan en estrategias complementarias llevan a cabo las actividades fuera del establecimiento educacional, principalmente en la consulta particular.

Los TTOO que se desempeñan en ambas estrategias realizan actividades grupales; los TTOO que trabajan en estrategias complementarias llevan a cabo talleres de habilidades funcionales, talleres grupales desde la perspectiva de Integración Sensorial y talleres preventivos según perfil psicosocial del niño. Además, los TTOO de ambas estrategias señalan colaborar en adaptaciones curriculares y trabajo administrativo y en menor medida, participan en consejo de profesores e investigación.

En cuanto a los profesionales con los que se relacionan (ver gráfico 4) los TTOO que trabajan en PIE señalan preferentemente a Psicólogo y Educador Diferencial. Por su parte, los TTOO que trabajan en estrategias complementarias mencionan relacionarse en distinto porcentaje con todos los integrantes del equipo multiprofesional señalado 
por el Ministerio de Educación (Psicólogo, Fonoaudiólogo, Psicopedagogo, Asistente Social). Es importante señalar que la modalidad de trabajo de los TTOO que se desempeñan en ambas estrategias es de tipo multidisciplinaria.

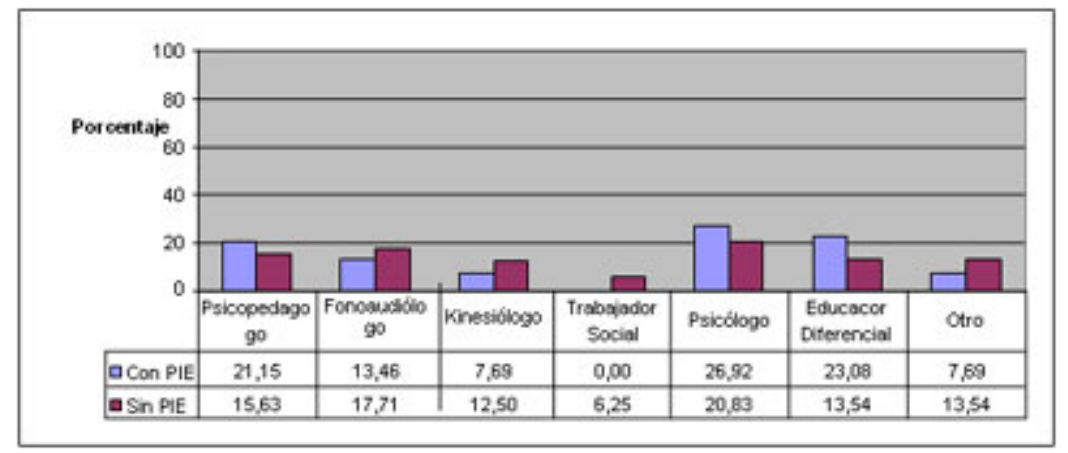

Figura 4: Distribución porcentual de TTOO según lugar de trabajo y profesional con que se relaciona

\section{- Discusión}

Los resultados demográficos obtenidos, la prevalencia de TTOO de sexo femenino se relacionan con antecedentes que señalan que Al inicio de la carrera se promovía sólo el ingreso de mujeres; actualmente ingresan hombres y mujeres, aunque se mantiene la tendencia de mayor egreso de estudiantes de sexo femenino, según lo que indican los Anuarios Estadísticos del Consejo de Rectores de las Universidades Chilenas. En cuanto a la Universidad de egreso, los resultados se relacionan con el breve período de implementación de la carrera en la Universidad de Playa Ancha, donde los registros indican que en el año 2002 se titulan los primeros TTOO.

Con respecto a las áreas de perfeccionamiento, los datos recopilados se relacionar con que los dos principales instancias de convocatoria de TTOO que participaron en el estudio son instancias de perfeccionamiento sobre Educación (Congreso de Profesionales no Docentes de Educación - APRONDE) e integración sensorial (Congreso latinoamericano de Integración Sensorial).

Es posible inferir que una de las razones del escaso índice de publicaciones puede ser la baja experiencia profesional de los TTOO en el sistema educativo regular.

En cuanto a las condiciones laborales, la mayoría los TTOO que se desempeñan en PIE son contratados por la Corporación Municipal y una escasa cantidad por el Departamento de Educación Municipal, lo que se puede comprender al considerar que el TO no es un profesional que conforme los equipos multiprofesionales de dicho departamento, por lo que no existen registros de los TTOO que trabajan en ellos.

La población que atienden los TTOO que trabajan en PIE se relaciona con los objetivos de esta estrategia; sin embargo, esto puede influir en que los TTOO no participen en diagnóstico de NEE de otros alumnos del establecimiento, disminuye la posibilidad del trabajo colaborativo con el resto del equipo profesional, además de aumentar el desconocimiento de la intervención del TO. En cuanto a los TTOO que se desempeñan en estrategias complementarias, estos trabajan con la mayor parte de la comunidad y 
con estudiantes con NEE asociadas o no a discapacidad, lo que responde a enfoques más inclusivos.

En relación a los niveles de educación, los TTOO que trabajan en PIE y en estrategias complementarias se desempeñan principalmente en nivel educativo básico la edad de los alumnos se relaciona con el nivel educativo que cursan. Los datos recopilados permiten discutir sobre la posibilidad de intervención del TO en el nivel prebásico, siendo esta la principal población objetivo del proceso de integración educativa debido a las proyecciones que esto brinda para el acceso, la mantención y el egreso del sistema educativo.

Los resultados obtenidos con respecto al fundamento teórico, estos se relaciona con que la mayor parte de la muestra que participa del estudio fue convocada en instancias de capacitación sobre teoría de Integración Sensorial.

En cuanto a las evaluaciones utilizadas, es importante señalar que los resultados se basan en el criterio utilizado por los TTOO para clasificar los instrumentos de evaluación como estandarizados o no estandarizados, siendo la observación clínica del desempeño ocupacional en contextos habituales la más referida por TTOO de ambas estrategias. Esto confirma la necesidad de unificar la terminología para realizar observación clínica y crear instrumentos que permitan validar el proceso de Terapia Ocupacional basada en la evidencia apoyada en la medición de resultados obtenidos.

El componente del ambiente físico sobre el que más intervienen los TTOO es el mobiliario y, en menor grado los objetos, aspectos en los que no se necesitan muchos recursos económicos para generar los cambios necesarios. En cuanto a la infraestructura, todos los TTOO realizan mayoritariamente evaluación, debido a que las posibilidades de cambio generalmente requieren intervenciones a largo plazo, mayores recursos económicos, lo que también se menciona como dificultad en la intervención.

Los TTOO utilizan sala de clases común y aula de recursos para realizar actividades individuales, preferentemente evaluación, lo que justificaría su participación en el equipo multiprofesional del Ministerio de Educación. En el trabajo en sala de clases el estudiante cuenta con el apoyo directo durante su desempeño ocupacional, pero puede generar cierta dependencia, sin embargo, es una buena posibilidad para evaluar el real desempeño ocupacional y las relaciones que establece con sus compañeros. Por otra parte, existe dificultad para realizar las actividades de una forma que no perjudique el desempeño de la actividad docente. En el aula de recursos, el TO puede apoyar aquellos aspectos más débiles del estudiante, pero este tipo de actividades no se realiza en el contexto de desempeño real, además, muchos establecimientos no cuentan con esta infraestructura.

EI PIE no plantea estrategias de intervención grupal, a diferencia de los programas gubernamentales y otras experiencias complementarias que si lo consideran; aun así, los TTOO que trabajan en PIE señalan esta modalidad utilizando las mismas dependencias consideradas en intervención individual, más otros espacios dentro y fuera del establecimiento, sin incluir la sala de clases. Los TTOO de ambas estrategias realizan otras actividades, que se relaciona con el Perfil Ocupacional por Competencias, elaborado por el Colegio de Terapeutas Ocupacionales de Chile.

Los TTOO se relacionan con profesionales de distintas áreas, principalmente con aquellos incluidos en el equipo multiprofesional descrito por el Ministerio de Educación. 


\section{Conclusiones}

En relación a las preguntas investigativas y los objetivos planteados, es posible concluir que existen TTOO apoyando el proceso de integración educativa en las regiones Quinta y Metropolitana, desde PIE y estrategias complementarias, presentando diferencias en las características de la práctica profesional.

Dada las limitaciones del estudio, no es posible determinar las características demográficas y de formación profesional. Los TTOO son titulados tanto de la Universidad de Chile y de la Universidad de Playa Ancha de Ciencias de la Educación, realizan perfeccionamientos en diversas áreas y la mayoría de ellos no realiza publicaciones.

En relación a la práctica profesional, existen diferencias entre ambas modalidades, en cuanto a condiciones laborales; población intervenida y lugar de intervención Se aprecian similitudes en relación al nivel educativo que intervienen, marcos teóricos que sustentan sus intervenciones y proceso de Terapia Ocupacional.

En ambas estrategias los TTOO evalúan diferentes ámbitos del desempeño ocupacional utilizando preferentemente evaluaciones no estandarizadas. La intervención incluye diferentes actividades en ambiente físico y social, con enfoque individual y/o grupal, participan en trabajo administrativo, adaptaciones curriculares, gestión e investigación.

Los TTOO que se desempeñan en PIE y en estrategias complementarias se relacionan con otros profesionales en modalidad multidisciplinaria, aún cuando no pertenezca al equipo de apoyo del establecimiento.

Los resultados concuerdan con los planteamientos del Colegio de Terapeutas Ocupacionales de Chile; la visión y práctica profesional del TO coincide con la visión del Humanismo y el Constructivismo. El primero al respetar y valorar la diversidad, las potencialidades, iniciativas e intereses de cada alumno y la influencia del medio ambiente (colegio) en la promoción y facilitación de los aprendizajes. El segundo al reconocer al estudiante como un ser activo y protagonista en el proceso educativo, que logra el aprendizaje por medio de la actividad y el contenido significativo de ella. Considerando en este proceso la facilitación tanto del ambiente físico como del social. Los TO del estudio apoyan efectivamente los procesos de integración educativa y además realizan acciones inclusivas.

Se sugiere aumentar estrategias de intervención grupal sin disminuir calidad de la intervención, mejorar sistematización y publicaciones de experiencias, junto con promover organización de TTOO que trabajan en el área.

Entre las proyecciones de este estudio se considera ser fuente de nuevas investigaciones y publicaciones, aportar en la formación de profesionales de pregrado con visión integradora - inclusiva, posible programa de post-grado para TO que trabajan en el sistema educativo regular; publicación en revistas especializadas de Educación y Terapia Ocupacional; además de contribuir a definir el perfil por competencias de TO en el área educativa. 


\section{- Agradecimientos}

- Terapeutas Ocupacionales de las regiones Quinta y Metropolitana que comparten experiencias apoyando procesos de integración educativa al Sistema Educativo Regular.

- Colegio de Terapeutas Ocupacionales de Chile, A.G.

- Corporación Chilena de Integración Sensorial

- Asociación de Profesionales no Docentes de Educación (APRONDE)

- Carmen Gloria de las Heras, Terapeuta Ocupacional, Autoridad Académica del Modelo de Ocupación Humana en Latinoamérica.

- Para mayor información contactarse a to educacion@yahoo.es

\section{- Referencias}

(1) Aguilar M. Síntesis en Español del Informe Warnock, 1991. Disponible en: http//didac.unizar.es/jlbernalwarnock.html, (consultado el 23 de junio de 2005).

(2) Norma DTO-830 Promulga Convención sobre Los Derechos del Niño, adoptada en la Asamblea general de las Naciones Unidas el 20 de noviembre de 1989 y suscrita por Chile el 26 de enero de 1990.

(3) UNESCO. Declaración de Salamanca y Marco de Acción para las Necesidades Educativas Especiales. Salamanca, España, 1994.

(4) Decreto Supremo de Educación No 490/90. Diario Oficial de la República de Chile, Santiago, Chile, 1990.

(5) Ley 19.284. Diario Oficial de la República de Chile, Santiago, Chile, 5 de enero de 1994.

(6) Decreto Supremo de Educación No 01/98. Diario Oficial de la República de Chile, Santiago, Chile, 11 de febrero de 2000.

(7) Consultora de Estudios, Asesorías y Planificación en Desarrollo Local - CEAS Ltda. Estudio a Nivel Muestral de la Calidad del Proceso de Integración Educativa. Disponible en:

http://www.mineduc.cl/biblio/documento/1433 ResumenejecutivoEstudioMues tral.pdf (consultado el 15 de junio de 2005)

(8) Gobierno de Chile \& FONADIS. Primer Estudio de la Discapacidad en Chile: ENDISC - CIF. Santiago, Chile: FONADIS, 2004. 
(9) Ministerio de Educación. Política Nacional de Educación Especial: Nuestro Compromiso con la Diversidad, capítulo 3, página 35. Santiago, Chile, 2005.

(10) Flores, R. Perfil por Ocupacional por Competencias del Terapeuta Ocupacional. Santiago, Chile, 2005.

(11) The American Occupational Therapy Association. Occupational therapy practice framework: Domain and Process. American Journal of Therapy. 56, 609 - 639, Pennsylvania. U.S.A., 2002.

(12) Consejo de Rectores Universidades Chilenas. Anuario Estadístico. Santiago, Chile: Secretaria General del Consejo de Rectores, 1962 a 1980 y 1981 a 2004. 